\title{
Dynamic Handoff Ordering Adjustment for Multimedia Cellular Network
}

\author{
Chow-Sing Lin and Pin-Ching Huang \\ Department of Information Management \\ Southern Taiwan University of Technology \\ Tainan, Taiwan (R.O.C.) \\ Email: \{mikelin,kid\}@msrg.mis.stut.edu.tw
}

\begin{abstract}
In multimedia cellular network, a Mobile Host (MH) requests multimedia services and may experience handoffs to several cells. When the target cell cannot provide adequate bandwidth for a service, instead of directly dropping a request, the $\mathrm{MH}$ is put into the handoff queue and hopefully the request bandwidth can be satisfied by later released bandwidth. Obviously, it is important to properly assign priorities for queued handoffing MHs based on their inborn dynamics to avoid unnecessary dropping. In this paper, we present a dynamic handoff priority adjustment (DHPA) scheme which applies handoff queuing scheme to order handoff requests and adjust handoff priority based on receiving signal strength, service class, and mobility of MHs. The goal of DHPA is to reduce call dropping probability to maintain QoS for users and also increase bandwidth utilization of multimedia wireless network.
\end{abstract}

Keywords: Multimedia wireless network, quality of service, handoff ordering, handoff queue.

\section{Introduction}

In recent years, due to the rapid advances in networking technology, providing multimedia service on cellular network now becomes feasible. The types of accessible multimedia contents, such as data, voice, music, and video have grown explosively [1][2][3][4]. On multimedia wireless network, MHs require various types of service. Each service requires different quality-of-service (QoS). Due to the mobility of MHs, how to maintain a consistent QoS of MHs become more difficult and challenging.

The typical infrastructure of cellular wireless network is made of several hexagons, called cells. MHs in a cell are served by a base station (BS). Between cell and cell there is an overlap area, and MHs in overlap area can receive signal from both BS.
When the Received Signal Strength (RSS) of the target cell reaches a certain threshold, the $\mathrm{MH}$ is handoffed to the target cell to continue the service.

When a MH enters the handoff zone, it issues a handoff request to the target cell. If the target cell cannot provide adequate bandwidth for on-going services either by bandwidth reservation scheme [7] or by guard channel scheme [10], instead of directly dropping the request, the $\mathrm{MH}$ is put into the handoff queue, and hopefully the requested bandwidth can be satisfied by later released bandwidth. Obviously, it is important to properly assign priorities for queued handoffing MHs based on their inborn dynamics to avoid unnecessary dropping. Particularly, the mobility of a MH may change erratically. The priority of a MH in handoff queue must be dynamically adjusted based on its mobility to reflect the urgency of handoff.

In this paper, we propose a dynamic handoff priority adjustment (DHAP) scheme to order queued handoffing requests of MHs. The mobility of a MH is classified as two modes, active mode and inactive mode, to specify whether a $\mathrm{MH}$ is continuously moving and rarely moved based on a threshold of the moving speed, respectively. In addition, the DHAP consists of multiple handoff queues, which are active handoff queue and inactive handoff queue. Handoffing MHs in active mode and inactive mode failing acquiring adequate bandwidth in target cell are inserted into active handoff queue and inactive handoff queue, respectively. The priority of a $\mathrm{MH}$ in active handoff queue is periodically adjusted by its dwelling time which indicates how soon the $\mathrm{MH}$ is to be handoffed to the target cell. On the other hand, the priority of a $\mathrm{MH}$ in inactive handoff queue is determined by service class and receive signal strength. The migration between active and inactive handoff queues may happen when the mobility of a queued handoffing $\mathrm{MH}$ changes.

The reminder of this paper is organized as follows. In section 2, related works are investigated. DHPA is presented in section 3. Section 4 presents simulation 
results and analysis. Finally, concluding remarks are given in section 5 .

\section{Related Work}

The simplest strategy of determining priority for a queued $\mathrm{MH}$ is based on its arriving order, such as First-In-First-Out (FIFO). Without considering other factors, FIFO cannot truly reflect the urgency of handoff due the erratic mobility of a $\mathrm{MH}$. Qian and Feng [8] present the minimum-dwelling-time scheme to prioritize handoff request. The priority of handoff request in queue is determined by its dwelling time in the handoff area. A measurement-based prioritizing scheme (MBPS) [5] has been developed for prioritizing the queue instead of the first in first out (FIFO). In MBPS, the priority is determined only based on the RSS of a MH. Ebersman and Tonguz [9] proposed a signal prediction priority queuing (SPPQ) using not only RSS but also moving speed which is interpreted as the change of RSS ( $\Delta$ RSS) to determine the priority of a MH. However, in SPPQ only one type of service is considered. Chang and Leu [6] further extended SPPQ algorithm, called Signal Strength for Multimedia Communications (SSMC) to handle the multiple types of multimedia traffic. In SSMC, the service class, RSS and $\triangle$ RSS are considered to determine the priority of a queued handoffing $\mathrm{MH}$. It fails to consider the situation when a $\mathrm{MH}$ stops moving or is even moving backward.

In this paper, we present a Dynamic Handoff Priority Adjustment (DHPA) scheme to extend SSMC scheme. In DHPA, we also use RSS, $\triangle$ RSS, and service class to determine the priority of a queued handoffing $\mathrm{MH}$. In addition, DHPA scheme further monitors the mobility of queued handoffing $\mathrm{MHs}$ to dynamically adjust handoff priorities.

\section{The Description of Dynamic Handoff Priority Adjustment}

\subsection{System Model of DHPA}

In DHPA, depending on the change of RSS, the mobility of a $\mathrm{MH}$ is classified as active mode or inactive mode. We also denote multiple handoff queues in the target cell. One is called active handoff queue, and the other is called inactive handoff queue. MHs failing acquiring bandwidth from the target cell in the handoff area are inserted into the active and inactive handoff queue based on their mobility, accordingly. The handoff priorities of the queued handoffing MHs are periodically adjusted also based on their mobility in the handoff area. When there are bandwidths released in the target cell, MHs in the active handoff queue are serviced first, and then those

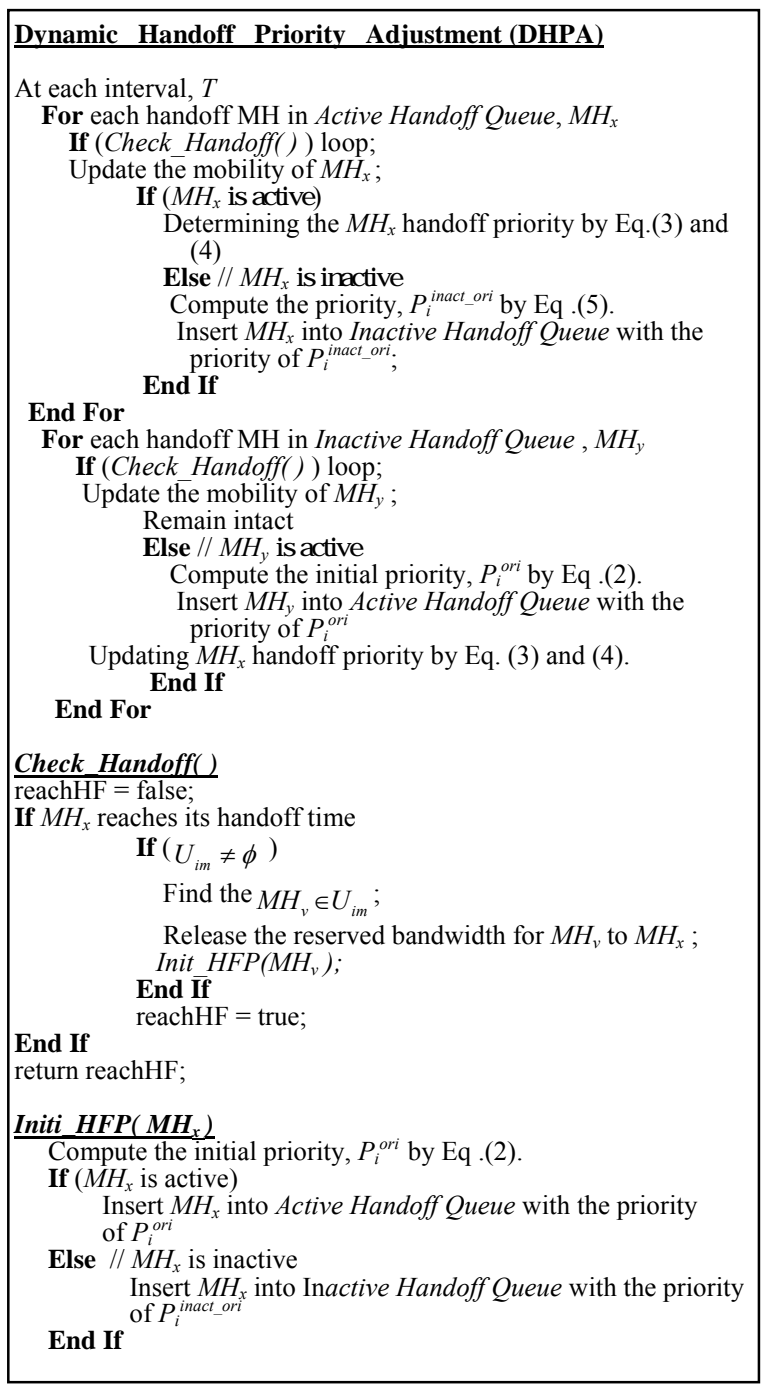

Fig. 1: The pseudo code of DHPA

in the inactive handoff queue if any bandwidth left.

In the paper, the mobility of a MH is classified as two modes, active and inactive, and it is updated periodically. We use (1) to define the mobility of a MH.

Mobility $=\left\{\begin{array}{c}\text { active, if }\left|\Delta R S S_{i}^{t}\right| \times w_{1}+\left|\Delta R S S_{i}^{t-1}\right| \times w_{2} \geq \delta \\ \text { inactive, if }\left|\Delta R S S_{i}^{t}\right| \times w_{1}+\left|\Delta R S S_{i}^{t-1}\right| \times w_{2}<\delta\end{array}\right.$

Assume $R S S_{i}^{t}$ and $R S S_{i}^{t-1}$ denote the RSS of a $\mathrm{MH}$ at time $t$ and time $t-1$, respectively. $\delta$ denotes the threshold of mobility. If the $|\triangle R S S|$ of a $\mathrm{MH}$ is larger then $\delta$, it means that it is keeping moving at reasonable speed. Its mobility is classified as active mode. In contrast, if the $|\triangle R S S|$ of a $\mathrm{MH}$ is less then $\delta$, it means that it is rarely moved or even stopped, and can be regarded as in inactive mode. In addition, when a $\mathrm{MH}$ moves out of handoff zone, the handoff request is no longer valid and must be moved out the handoff queue. 


\subsection{Dynamic Handoff Priority Adjustment}

When a MH enters the handoff zone, it issues a handoff request to the target cell. If the target cell cannot provide adequate bandwidth for requested service, the $\mathrm{MH}$ is put into the handoff queue, and hopefully the requested bandwidth can be satisfied by later released bandwidth. Before inserting the $M H_{i}$ into the handoff queue, the initial priority, $P_{i}^{\text {ori }}$, is computed by using (2), where $S C_{i}, \Delta R S S_{i}$, and $R S S_{i}$ denote the priority of requested service class, the change of received signal strength, and received signal strength of $M H_{i}$, respectively.

$$
P_{i}^{o r i}=S C_{i} \times\left|\Delta R S S_{i}\right| \times \frac{1}{R S S_{i}}
$$

Then, the mobility of $M H_{i}$, is determined by (1). If $M H_{i}$ is in active mode, it is inserted into active handoff queue; if $M H_{i}$ is in inactive mode, it is inserted into inactive handoff queue. To timely reflect the erratic mobility of a $\mathrm{MH}$, its priority needs to be periodically adjusted.

If a MH keeps moving in handoff area and stays in active mode, the DHPA scheme periodically updates its handoff priority according to the dwelling time which indicates how soon a $\mathrm{MH}$ is expected to be handoffed to the target cell. Intuitively, a $\mathrm{MH}$ which has shorter dwelling time should have higher probability to handoff into the target cell, and thus should be given a higher handoff priority. Assume $D T_{i}^{t}$ denotes the dwelling time of $M H_{i}$ in the handoff area, $R S S_{i}^{\text {handoff }}$ denotes the threshold of receive signal strength to handoff, $w_{1}$ and $w_{2}$ denote the relative weight of $R S S_{i}^{t}$ and $R S S_{i}^{t-1}$. Then, the dwelling time of $M H_{i}$ in the active handoff queue can be estimated as (3). Based on the dwelling time of $M H_{i}$, we use (4) to periodically update its handoff priority, $P_{i}^{\text {active }}$

$$
\begin{gathered}
D T_{i}^{t}=\frac{R S S_{i}^{t}-R S S_{i}^{\text {handoff }}}{\left|\Delta R S S_{i}^{t}\right| \times w_{1}+\left|\Delta R S S_{i}^{t-1}\right| \times w_{2}} \\
P_{i}^{t}=P_{i}^{t}+P_{i}^{t-1} \times \frac{1}{D T_{i}^{t}}
\end{gathered}
$$

On the other hand, if MHs are rarely moved, they are classified as in inactive mode, and thus are inserted into inactive handoff queue. Assume that $R S S_{i}^{\text {issue_handoff }}$ denotes the threshold of the RSS to issue a handoff request, and the handoff priority of a $\mathrm{MH}$ in inactive mode, $P_{i}^{\text {inact_ori }}$, can be calculated by (5).

$$
P_{i}^{\text {inact_ori }}=S C_{i} \times\left(\frac{R S S_{i}^{\text {issue_handoff }}}{R S S_{i}^{t}}-1\right)
$$

MHs in active and inactive handoff queue may migrate to each other when their mobilities change. When a MH migrates to the other queue, its priority is initialized by using (2) and (5). In addition, if a $\mathrm{MH}$, no matter it is in active or inactive mode, is moving away from the target cell and is out of the handoff zone, it is removed from the handoff queue. Furthermore, In DHPA, when a MH who successfully reserves bandwidth in the target cell turns into inactive mode, i.e., the reserved bandwidth may not be used for a while, it is added into so-called immobile set, $U_{i m}$. MHs in the $U_{i m}$ may be asked to release the reserved bandwidth in order to save a dropping handoff call. Figure 1 shows the pseudo code of DHPA.

\section{Simulation Results}

The simulation model is assumed on a multimedia cellular network, which consists of 25 cells. The area of one cell is $4 * 4 \mathrm{~km}^{2}$. Each cell has 50 channels, and each channel has the network capacity of $64 \mathrm{kbps}$. There are three service classes in the simulation [6]. The call arrival rate follows Poisson distribution, and MHs are evenly distributed to 25 cells. Each $\mathrm{MH}$ randomly selects a moving direction $[0,360]$ and speed $[30 \mathrm{~km} \sim 90 \mathrm{~km}] . w_{1}$ and $w_{2}$ is equal to 0.7 and 0.3 , respectively. Threshold of mobility, $\delta$ is 0.1 . At each interval a MH has 0.85 probabilities staying in current moving speed. In addition, it also has 0.02 probabilities moving away from the target cell.

In simulation we investigate the performance of DHPA in terms of the call blocking probability (CBP), call dropping probability (CDP), and bandwidth utilization $(\mathrm{BU})$ by comparing with other ordering strategies, such as first-in-first-out (FIFO), and signal strength for multimedia communication (SSMC). In FIFO, we assume that if there are no channels left, new calls are simply blocked and handoff requests are queued in a handoff queue.

Figure 3 shows the CBP of FIFO, SSMC, and DHPA with respect to the increase of call arrival rate. Because all the three approaches give higher priority to handoff calls than to new calls on bandwidth allocation, they perform almost equally well. Figure 4 shows the CDP of FIFO, SSMC, and DHPA with respect to the increase of call arrival rate. The CDP of our proposed DHPA obviously is much lower than that of FIFO and SSMC on average by $20 \%$ and $17 \%$, respectively. There are two key characteristics revealing the superiority of DHPA in CDP. First, the priority of active handoff queue is adjusted mostly based on the dwelling time which is more suitable to reflect the urgency of handoff. Second, with DHPA bandwidth successfully reserved by inactive handoff calls may not be used for a while, and it is released to save dropping handoff calls. In this way, the CDP can be further reduced. Figure 5 shows the bandwidth 
utilization of FIFO, SSMC, and DHPA with respect to the increase of call arrival rate. Obviously, our proposed DHPA scheme has the best bandwidth utilization. This is because with DHPA, again, bandwidth successfully reserved by inactive handoff calls can be borrowed to save dropping handoff calls. As a result, the idleness of bandwidth is reduced, and thus the bandwidth utilization of DHPA is better than that of FIFO and SSMC.

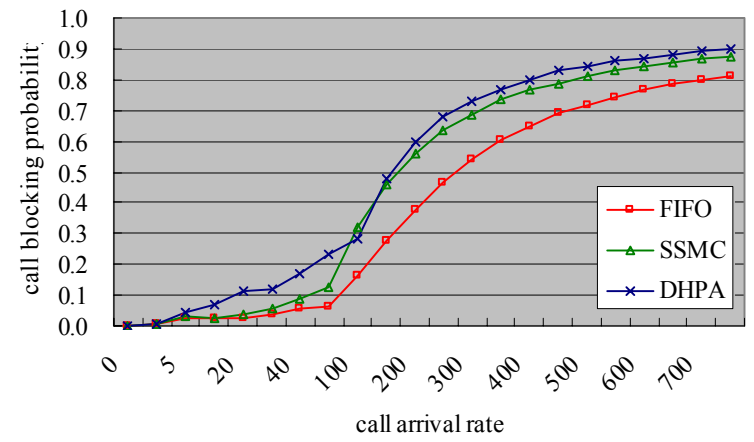

Fig. 3: Call blocking probability

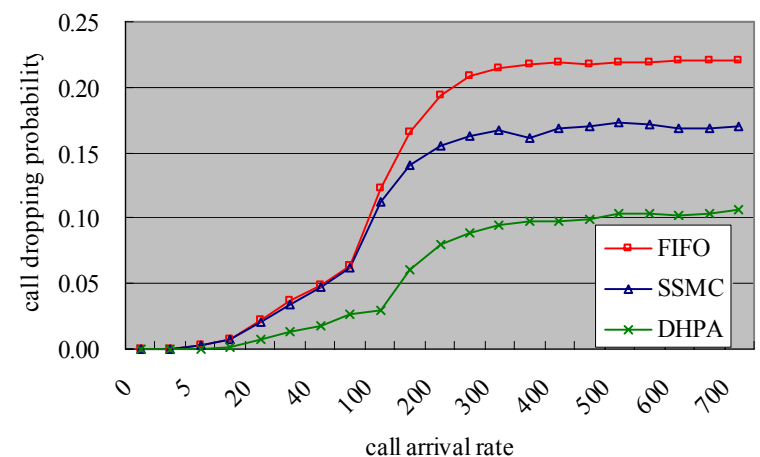

Fig. 4: Call dropping probability

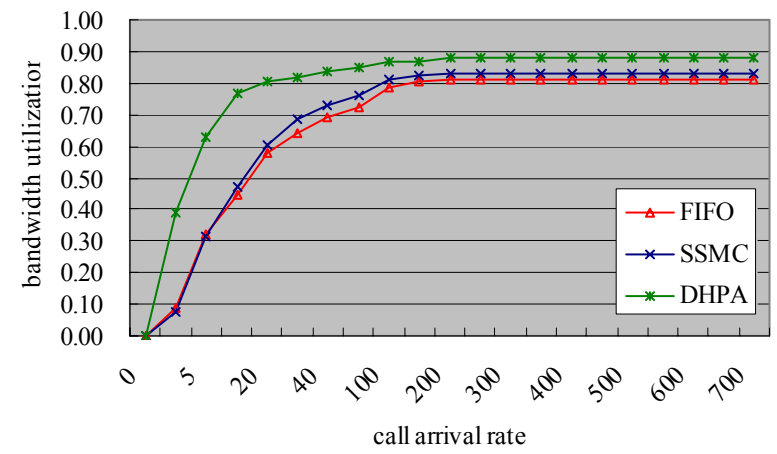

Fig. 5: Bandwidth utilization

\section{Concluding Remarks}

When the target cell cannot provide adequate bandwidth for a handoff call, instead of dropping the request, queuing approach is the major technique to save dropping handoff MHs. How to effectively and dynamically reflect the urgency of queued handoff
MHs is very crucial to maintain the QoS of a MH. In this paper, we propose the DHPA scheme which utilizes the mobility of MHs to dynamically adjust handoff priority to reduce the CDP and increase bandwidth utilization as well. The simulations show positive results on the proposed DHPA scheme.

\section{References}

[1] M. El-Kadi, S. Olariu, and H. Abdel-Wahab, "A Rate-Based Borrowing Scheme for QoS Provisioning in Multimedia Wireless Networks," IEEE Transactions on Parallel and Distributed Systems, pp.156-166, 2002.

[2] B. M. Epstein and M. Schwartz, "Predictive Qos Based Admission Control for Multiclass Traffic in Cellular Wireless Networks," IEEE Journal on Selected Areas in Communications, pp.523-534, 2000.

[3] A. Mlla, M. El-Kadi, S. Olariu, and P. Todorova, "A Fair Resource Allocation Protocol for Multimedia Wireless Networks," IEEE Transactions on Parallel and Distributed Systems, pp.63-71, 2003.

[4] C. Oliverira, J.B. Kim, and T. Suda, "An Adaptive Bandwidth Reservation Scheme for Highspeed Multimedia Wireless Networks," IEEE Journal on Selected Areas in Communications, pp.858874, 1998.

[5] S. Tekiny and B. Jabbari, "A Measurement-Based Prioritization Scheme for Handovers in Mobile Cellular Networks," IEEE Journal on Selected Areas in Communications, pp.1343 - 1350, 1992.

[6] R.S. Chang and S.J. Leu, "Handoff Ordering Using Signal Strength for Multimedia Communications in Wireless Networks," IEEE Transactions on Wireless Communications, pp. $1526-1532,2004$.

[7] C. Oliverira, J.B. Kim, and T. Suda, "An Adaptive Bandwidth Reservation Scheme for High-speed Multimedia Wireless Networks," IEEE Journal of Selected Areas in Communications, pp. 858-874., 1998

[8] R. Quan and G. Feng, "A Minimum-Dwelling Time Prioritization Scheme for Handoff in Mobile Cellular Systems," International Conference on Communication Technology, 1998.

[9] H.G. Ebersman and O.K. Tonguz, "Handoff Ordering Using Signal Prediction Priority Queuing in Personal Communication Systems," IEEE Transactions on Vehicular Technology, pp.20 - 35, 1999

[10] H.H. Liu, "Guard channel sharing strategies in integrated voice/data mobile networks," Advanced Information Networking and Applications, pp.7982, 2004. 